\title{
Efecto de la aplicación de aire caliente humidificado como tecnología poscosecha en la calidad de la Feijoa
}

\section{Effect of application of hot air humidified as post- harvest technology in the quality of Feijoa}

David Leopoldo Sánchez-Rubio', Jhon Harrison Pérez-Benavides², Claudia Patricia Pérez-Rodríguez ${ }^{3}$

Sánchez-Rubio, D; Pérez-Benavides, J; Pérez-Rodríguez, C. Efecto de la aplicación de aire caliente humidificado como tecnología poscosecha en la calidad de la Feijoa. Tecnología en Marcha. Vol. 33-3. Julio-Setiembre 2020. Pág 57-67.

doi) https://doi.org/10.18845/tm.v33i3.4371

Fecha de recepción: 13 de agosto de 2019 Fecha de aprobación: 28 de noviembre de 2019

1 Estudiante de pregrado en Ingeniería Agrícola. Universidad Nacional de Colombia, Sede Bogotá; Facultad de Ingeniería; Departamento de Ingeniería Civil y Agrícola. Colombia. Correo electrónico: dalsanchezru@unal.edu.co

2 Estudiante de postgrado en Ingeniería Agrícola. Universidad Nacional de CoIombia, Sede Bogotá; Facultad de Ingeniería; Departamento de Ingeniería Civil y Agrícola. Colombia. Correo electrónico: jhperezb@unal.edu.co

3 Profesora asociada. Universidad Nacional de Colombia, Sede Bogotá; Facultad de Ingeniería; Departamento de Ingeniería Civil y Agrícola. Colombia. Correo electrónico: cpperezr@unal.edu.co 


\title{
Palabras clave
}

Acca sellowiana; tecnologías poscosecha; calidad.

\section{Resumen}

La feijoa (Acca sellowiana) es una fruta climatérica exótica de América del Sur, por su atractivo sabor agridulce se ha posicionado tanto en el mercado nacional como internacional. Por este motivo es pertinente avanzar en el desarrollo y aplicación de tecnologías de poscosecha que propendan por su adecuado manejo y conservación. Este estudio tuvo como objetivo evaluar el efecto en la calidad poscosecha de frutos de feijoa sometidos a una corriente de aire a temperatura de $41^{\circ} \mathrm{C}$ y HR de $57 \%$ por 2 horas (T1) y por 4 horas (T2). Posteriormente se evaluaron parámetro físico - químicos en el almacenamiento durante dos semanas en condiciones ambientales. Se encontró disminución en la pérdida de peso de los tratamientos frente a control, así como disminución en la contracción del diámetro ecuatorial sin detrimento de parámetros químicos como pH, acidez total titulable (ATT) e índice de madurez (IM). Entre los dos tratamientos, el que presenta mejores condiciones es el T2 $\left(41^{\circ} \mathrm{C}, 57 \% \mathrm{HR}\right.$, por cuatro horas) según parámetros como la pérdida de peso, contracción del diámetro ecuatorial, pH, ATT e IM.

\section{Keywords}

Acca sellowiana; post-harvest technologies; quality.

\begin{abstract}
The feijoa (Acca sellowiana) is an exotic climacteric fruit in South America, by its attractive sweet and sour flavor; it has been positioned in the market national as international. For this reason, is relevant to advance in the development and application of post-harvest technologies that foster their appropriate management and conservation. This study had as aim to evaluate the effect on postharvest quality of feijoa fruits subjected to a current air at temperature of $41^{\circ} \mathrm{C}$ and relative humidity of $57 \%$ for 2 hours (T1) and for 4 hours (T2). Subsequently, were evaluated physical and chemical parameters during the storage for two weeks in ambient conditions. Was found decrease in the weight loss of treatments in contrast with the control, also the decrease in contraction of the equatorial diameter without detriment of chemical parameters such as $\mathrm{pH}$, titrable total acidity (ATT) and maturity index (IM). Among the two treatments, the best one is $\mathrm{T} 1\left(41^{\circ} \mathrm{C}, 57 \% \mathrm{HR}\right.$, for two hours) depending on parameters such as the weight loss and contraction of the equatorial diameter, $\mathrm{pH}$, ATT and IM.
\end{abstract}

\section{Introducción}

La Feijoa (Acca sellowiana) pertenece a familia de las Mirtáceas junto con la guayaba. Es originaria del Sur de Brasil, Uruguay, Paraguay y Norte de Argentina. Este fruto es poco conocido en otros países y el que más lo produce para su comercialización es Nueva Zelanda en donde hay alrededor de 500 ha de cultivo. Este fruto puede ser consumido crudo o en dulces, los pétalos de sus flores también son comestibles en ensaladas [1]. Para la región andina colombiana la feijoa es un fruto por explotar para la exportación, presenta adaptabilidad de siembra en zonas con altitudes que comprenden entre los 1.800 a 2.700 msnm, por ejemplo. Es un fruto climatérico con tasas de respiración y producción de etileno bastante altas, por lo que, después de la cosecha el producto naturalmente disminuye considerablemente propiedades 
como el peso, pH y la turgencia [2], razón por la cual es pertinente encontrar tecnologías que permitan preservar la calidad del producto durante el almacenamiento y comercialización.

Estos frutos son llamativos por su elación de ácidos y azucares, por lo cual es importante definir un tratamiento que además de preservar la calidad del producto, permita preservar tales características. En oriente se ha investigado en el uso de tratamientos térmicos para la conservación de productos, alcanzando resultados satisfactorios, en la referencia [3] se indica que para durazno el uso de corrientes de aire humidificado mantuvo sus propiedades químicas y mecánicas, por otro lado, en la referencia [4] se reporta que para habichuela y frijol esta tecnología retrasó la pérdida de peso y preservó la apreciación del sabor y la textura. La implementación de corrientes de aire humidificado puede dar respuestas favorables a la resistencia de daño por frío y al control de la maduración [5].

Este estudio tiene como objetivo evaluar la respuesta en el almacenamiento de los frutos de feijoa al exponerse previamente a corrientes de aire humidificado, esta evaluación se realiza mediante el seguimiento de la pérdida de peso, propiedades mecánicas y fisicoquímicas, se espera que este trabajo siente bases para la implementación de tecnologías de bajo costo para estos productos.

\section{Metodología}

Esta investigación se realizó en el 2016 en el laboratorio de poscosecha del campus de la Universidad Nacional de Colombia, coordenadas 4³8'08"N 7404'58"O en la ciudad de Bogotá. Los frutos se cosecharon en su madurez fisiologica en el municipio de Tibasosa (Boyacá, Colombia) a $2.538 \mathrm{msnm}$. Las muestras fueron seleccionadas y clasificadas en un arreglo aleatorio, posteriormente fueron lavadas, desinfectadas con hipoclorito de sodio ( $\mathrm{NaClO})$ en una inmersión de 25 ppm, seguido de una inmersión en agua con el fin de eliminar los excesos de $\mathrm{NaClO}$.

Se establecieron tres tratamientos: a) Tratamiento control (TC), b) Tratamiento 1 (T1): Corriente de aire humidificado a $41^{\circ} \mathrm{C}$ y $57 \%$ de humedad relativa por 2 horas y c) Tratamiento 2 (T2): Corriente de aire humidificado a $41^{\circ} \mathrm{C}$ y $57 \%$ de humedad relativa por 4 horas. Los frutos de feijoa fueron dispuestos en bandejas enmalladas por donde se pasó la corriente de aire caliente humidificado en un psicrómetro, que permitía el control de la temperatura de bulbo seco y bulbo húmedo.

Posteriormente fueron almacenadas por 12 días a condiciones ambiente $\left(20^{\circ} \mathrm{C}\right.$ y $49 \%$ de humedad relativa) de humedad relativa. Se realizaron análisis de laboratorio de Porcentaje de pérdida de peso, índice de color interno (IC-I), índice de color externo (IC-E), porcentaje de contracción del diámetro ecuatorial, intensidad respiratoria (IR), carga máxima, deformación, trabajo, carga final, solidos solubles totales (SST), pH, acidez total titulable (ATT) e índice de madurez (IM), durante dos semanas, tomando datos por triplicado.

Los equipos usados para tomar las mediciones fueron (ver figura 1): Una balanza con precisión $0,001 \mathrm{Kg}$ para la pérdida de peso, la medición de los IC interno e IC externo se realizaron con un colorímetro digital "Konica Minolta CR-410" que tiene coordenadas del modelo cromático CIELab*, un pie de rey de 0,01 mm de precisión para determinar el diámetro ecuatorial, para la IR se usaron sensores infrarrojos "Vemier" en cámaras de respiración de 2L obteniéndose registros en ppm de la concentración de $\mathrm{CO} 2$, en cuanto a la consistencia para determinar la carga máxima (Kgf/cm2), deformación $(\mathrm{mm})$, trabajo $(\mathrm{mJ})$ y la carga final (gf) se midieron con un analizador de textura "Brookfield CT3", IOs SST ( ${ }^{\circ}$ Brix) se midieron con un refractómetro manual óptico "SO-RH", a través del método potenciométrico se midió pH y con un Hidróxido de Sodio a 0,1 $\mathrm{N}$ se midió ATT. 
El análisis estadístico de los datos se hizo a partir de matrices de correlación de Pearson, análisis de varianzas y medias, análisis de tendencias. Con estos métodos se nota la dependencia o variación de la información obtenida y la evaluación del comportamiento de los diferentes tratamientos.

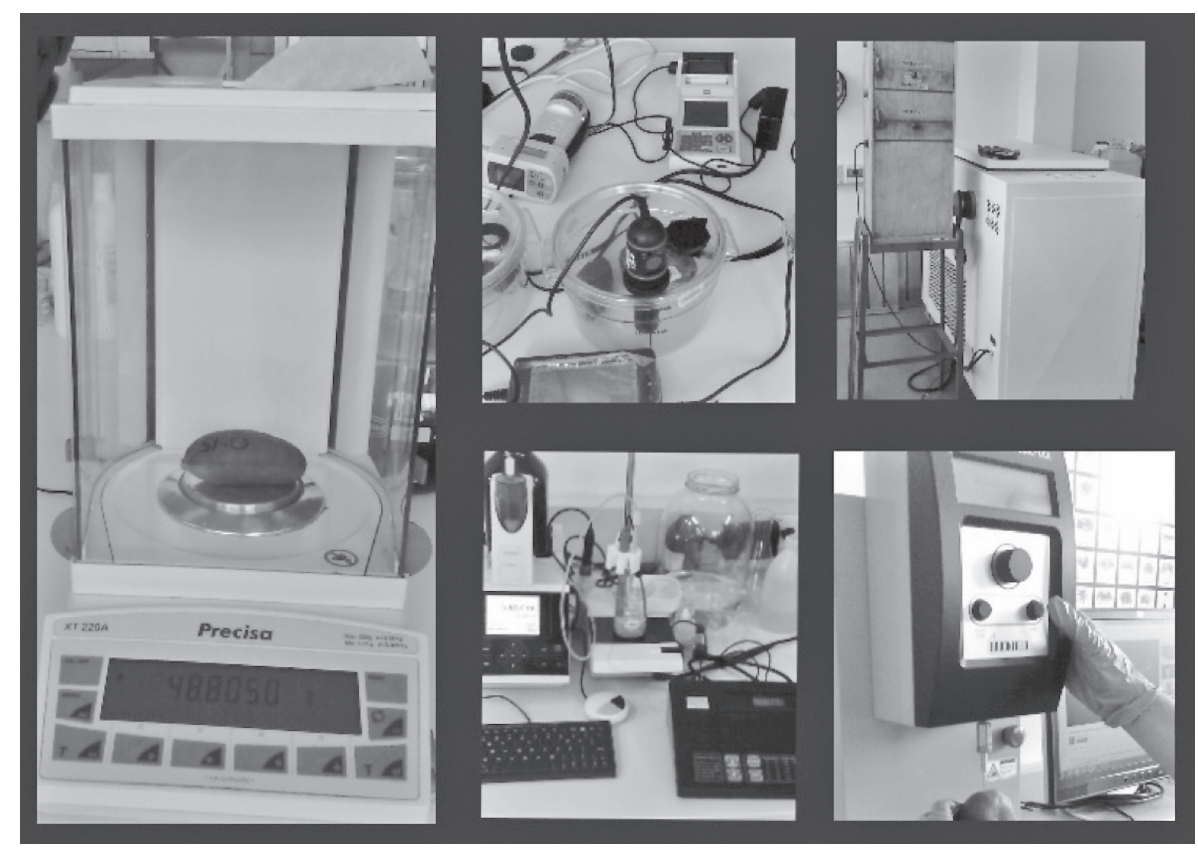

Figura 1. Foto de los equipos.

\section{Resultados y discusión}

Los datos presentaron los estadísticos descriptivos y correlaciones a través de la matriz de Pearson mostrados en los cuadros 1, 2, 3 y 4.

Cuadro 1. Estadísticos descriptivos

\begin{tabular}{|c|c|c|c|c|c|c|c|c|c|c|c|c|c|}
\hline & $\begin{array}{c}\text { \%Perdida de } \\
\text { peso }\end{array}$ & IC EXTERNO & $\begin{array}{c}\% \mathrm{DE} \\
\text { CONTRACCIÓN }\end{array}$ & TR & Peak Load & Deaf Peak & Work & Final Load & IC Interno & SST & $\mathrm{pH}$ & AT & IM \\
\hline \multicolumn{14}{|c|}{ CONTROL } \\
\hline Media & 10,09495267 & \begin{tabular}{|l|}
$-15,2562629$ \\
\end{tabular} & 4,320709348 & 75,8460882 & 29,7928769 & 4,42111111 & 33,952037 & 92,2962963 & $-0,36381336$ & 11,4111111 & 3,74027778 & 0,10255212 & 157,530471 \\
\hline DesvTipica & 7,206314049 & 1,01804754 & 2,75538204 & 38,9588604 & 7,8866629 & 1,05939069 & 7,09745197 & 24,5389755 & 1,93144392 & 1,01075696 & 0,64071697 & 0,07131662 & 87,6615634 \\
\hline Varianza & 51,93096217 & 1,03642079 & 7,592130187 & 1517,7928 & 62,1994518 & 1,12230864 & 50,3738245 & 602,161317 & 3,7304756 & 1,02162963 & 0,41051824 & 0,00508606 & 7684,5497 \\
\hline \multicolumn{14}{|c|}{ TRATAMIENTO 1} \\
\hline Media & 3227127 & $-12,6246225$ & 572532056 & 66,796926 & 31,6000245 & 4,67916667 & 33,4311111 & 89,8842593 & 0,00112614 & 11,3083333 & 3,80111111 & 0,09646871 & 153,082903 \\
\hline DesvTipica & 074681 & 1,55763041 & 5216 & 49,4795549 & 9,45145663 & 0,90748219 & 6,83952157 & 63259 & 55916 & 31897 & 0,43437141 & 0,045243 & 101,156314 \\
\hline Varianza & 37,528791 & 2,4262125 & 5,726856849 & 2448,22635 & 89,3300324 & 0,82352392 & 46,7790553 & 1053,41309 & 2,48995511 & 0,47930556 & 0,18867852 & 0,00204693 & 10232,5999 \\
\hline \multicolumn{14}{|c|}{ TRATAMIENTO 2} \\
\hline Media & 8,121715587 & $-15,6855999$ & 3,051363516 & 74,8227386 & 28,4252413 & 4,98518519 & 30,3768519 & 84,7314815 & 1,33249245 & 11,125 & 3,88694444 & 0,07492682 & 157,800608 \\
\hline \begin{tabular}{|l|} 
DesvTipica \\
\end{tabular} & 6,161084055 & 1,22320759 & 2,334486377 & 56,4660163 & 2,95136807 & 0,9525707 & 2,14460465 & 35,8822262 & 1,92845607 & 1,10281206 & 0,37321935 & 0,02451027 & 36,339520 \\
\hline Varianza & 37,95895674 & 1,49623681 & 5,449826644 & 3188,411 & 8,71057348 & 0,90739095 & 4,59932909 & 1287,53416 & 3,71894282 & 1,21619444 & 0,13929269 & 0,00060075 & 1320,56072 \\
\hline
\end{tabular}


Cuadro 2. Matriz de Pearson tratamiento control

\begin{tabular}{|c|c|c|c|c|c|c|c|c|c|c|c|c|c|}
\hline \multicolumn{14}{|c|}{ CONTROL } \\
\hline \multicolumn{14}{|c|}{ Correlaciones } \\
\hline & Pérdida Peso & IC-E & Contracción & $\mathbb{R}$ & Carga Máx & Deformación & Trabajo & Carga Final & IC-I & SST & $\mathrm{pH}$ & ATT & IM \\
\hline Pérdida Peso & 1 & & & & & & & & & & & & \\
\hline IC-E & $816^{\circ}$ & 1 & & & & & & & & & & & \\
\hline Contracción & ,967" & ,667 & 1 & & & & & & & & & & \\
\hline $\mathbb{R}$ & ,301 & ,122 & ,228 & 1 & & & & & & & & & \\
\hline \begin{tabular}{|l|} 
Carga Máx \\
\end{tabular} &,$- 916^{\circ}$ & $-6,620$ &,$- 934 "$ &,- 157 & 1 & & & & & & & & \\
\hline \begin{tabular}{|l|} 
Deformación \\
\end{tabular} & ,968" & ,731 & ,931" & ,487 &,$- 906^{\circ}$ & 1 & & & & & & & \\
\hline Trabajo & $\begin{array}{l}-, 789 \\
-78\end{array}$ &,- 399 &,$- 902^{\circ}$ &,- 032 & ,868" &,- 770 & 1 & & & & & & \\
\hline Carga Final &,- 765 &,- 544 &,$- 815^{\circ}$ &,- 186 & ,674 &,- 759 &, $869^{\circ}$ & 1 & & & & & \\
\hline IC-I & ,956" & ,929" &, $862^{\circ}$ & ,286 & -801 &, $896^{\circ}$ &,- 589 & $\begin{array}{l}-, 627 \\
-67\end{array}$ & 1 & & & & \\
\hline SST & -365 &,- 209 & $\begin{array}{l}-, 504 \\
-54\end{array}$ & ,467 & ,394 &,- 257 & ,741 & ,751 &,- 212 & 1 & & & \\
\hline $\mathrm{pH}$ & ,919" & ,621 &, $925^{\prime \prime \prime}$ & ,231 &,- 974 & $902^{\circ}$ &,- 783 &,- 566 & $835^{\circ}$ &,- 247 & 1 & & \\
\hline ATT &,$- 928 "$ &,- 631 &,$- 980 "$ &,- 098 & ,937" &,$- 887^{\circ}$ & ,957" &, $864^{\circ}$ &,- 796 & ,637 &,$- 886^{\circ}$ & 1 & \\
\hline IM & ,973" & ,808 & ,953"' & ,300 &,$- 823^{\circ}$ & ,924" &,- 778 &,$- 831^{\circ}$ & ,936" &,- 449 &, $826^{\circ}$ &,$- 911^{\circ}$ & 1 \\
\hline \multirow{2}{*}{\multicolumn{14}{|c|}{${ }^{*}$. La correlación es significante al nivel 0,05 (bilateral). }} \\
\hline 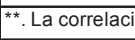 & & $\overline{0,0}$ & & & & & & & & & & & \\
\hline
\end{tabular}

Cuadro 3. Matriz de Pearson tratamiento 1

\begin{tabular}{|c|c|c|c|c|c|c|c|c|c|c|c|c|c|}
\hline \multicolumn{14}{|c|}{ TRATAMIENTO 1} \\
\hline \multicolumn{14}{|c|}{ Correlaciones } \\
\hline & Pérdida Peso & IC-E & Contracción & $\mathrm{IR}$ & Carga Máx & Deformación & Trabajo & \begin{tabular}{|l} 
Carga Final \\
\end{tabular} & IC-I & SST & $\mathrm{pH}$ & ATT & IM \\
\hline Perdida Peso & 1 & & & & & & & & & & & & \\
\hline IC-E &, $853^{\circ}$ & 1 & & & & & & & & & & & \\
\hline Contracción & ,987" & ,803 & 1 & & & & & & & & & & \\
\hline$\sqrt{\mathbb{R}}$ &,- 489 &,- 201 & $\begin{array}{c}-, 527 \\
\end{array}$ & 1 & & & & & & & & & \\
\hline \begin{tabular}{|l} 
Carga Máx \\
\end{tabular} &,$- 919 "$ &,- 630 &,$- 964 "$ & ,643 & 1 & & & & & & & & \\
\hline Deformación & ,924" & ,654 & ,944" &,- 713 &,- 969 & 1 & & & & & & & \\
\hline Trabajo &,- 771 &,- 553 &,- 807 & ,076 & ,778 &,- 646 & 1 & & & & & & \\
\hline Carga Final &,- 610 &,- 388 &,- 679 & ,025 & ,685 &,- 502 & ,960" & 1 & & & & & \\
\hline IC-I & ,987" &, $900^{\circ}$ &, $975^{\prime \prime}$ &,- 489 &,$- 889^{\circ}$ &, $887^{\circ}$ & $\begin{array}{l}-, 734 \\
\end{array}$ &,- 589 & 1 & & & & \\
\hline SST & $\begin{array}{c}-198 \\
-198\end{array}$ & $-1,162$ & $\begin{array}{ll}-, 058 \\
\end{array}$ &,- 050 & $\begin{array}{c}-, 047 \\
\end{array}$ &,- 121 &,- 045 & $\begin{array}{c}-279 \\
-29\end{array}$ &,- 119 & 1 & & & \\
\hline $\mathrm{pH}$ &, $852^{\circ}$ &, $871^{\circ}$ &, $881^{\circ}$ &,- 279 & $\begin{array}{c}-, 794 \\
\end{array}$ & ,714 &,- 759 &,- 703 &, $901^{\circ}$ & 253 & 1 & & \\
\hline ATT &,$- 981^{\prime \prime \prime}$ &,$- 916^{\circ}$ &,$- 958^{\prime \prime}$ & (349 &, $857^{\circ}$ &,$- 865^{\circ}$ & $\begin{array}{l}, 762 \\
\end{array}$ & ,589 &,$- 975^{\prime \prime}$ & 209 &,$- 879^{\circ}$ & 1 & \\
\hline IM &, $844^{\circ}$ & ,997" & ,799 &,- 237 & $\begin{array}{l}-, 634 \\
\end{array}$ & 664 &,- 517 &,- 355 &, $895^{\circ}$ &,- 124 &, $874^{*}$ &,$- 907^{\circ}$ & 1 \\
\hline
\end{tabular}

Cuadro 4. Matriz de Pearson tratamiento 2.

\begin{tabular}{|c|c|c|c|c|c|c|c|c|c|c|c|c|c|}
\hline \multicolumn{14}{|c|}{ TRATAMIENTO 2} \\
\hline \multicolumn{14}{|c|}{ Correlaciones } \\
\hline & \begin{tabular}{|l|} 
Pérdida Peso \\
\end{tabular} & IC-E & Contracción & IR & Carga Máx & Deformación & Trabajo & Carga Final & IC-I & SST & $\mathrm{pH}$ & ATT & IM \\
\hline Pérdida Peso & 1 & & & & & & & & & & & & \\
\hline IC-E &, $910^{\circ}$ & 1 & & & & & & & & & & & \\
\hline Contracción & $997^{\prime \prime \prime}$ & ,927" & 1 & & & & & & & & & & \\
\hline $\mathbb{R}$ &,- 501 &,- 352 & $\begin{array}{ll}-, 507 \\
\end{array}$ & 1 & & & & & & & & & \\
\hline Carga Máx &,$- 983^{* \prime}$ &,$- 940 "$ &,$- 979^{\prime \prime}$ & ,505 & 1 & & & & & & & & \\
\hline Deformación & ,513 & 184 & ,506 &,- 306 & -361 & 1 & & & & & & & \\
\hline Trabajo & $\begin{array}{l}-684 \\
-64\end{array}$ &,- 655 & $\begin{array}{l}-, 694 \\
\end{array}$ &, $915^{\circ}$ & ,731 &,- 129 & 1 & & & & & & \\
\hline \begin{tabular}{|l} 
Carga Final \\
\end{tabular} & ,368 & ,211 & ,397 &,- 627 & -247 & ,625 &,- 466 & 1 & & & & & \\
\hline IC-I & $905^{\circ}$ & $988^{\prime \prime}$ & ,921" &,- 422 &,- 950 " & 163 &,- 715 & ,173 & 1 & & & & \\
\hline SST &,$- 914^{\circ}$ & $\begin{array}{l}-690 \\
-690\end{array}$ &,$- 905^{\circ}$ & ,515 &, $846^{\circ}$ &,- 794 & ,553 & $\begin{array}{l}-, 466 \\
\end{array}$ & $-6,697$ & 1 & & & \\
\hline $\mathrm{pH}$ & ,550 & ,432 & ,534 & $\begin{array}{l}, 669 \\
\end{array}$ &,- 622 & ,170 &,- 713 & $\begin{array}{c}-, 045 \\
\end{array}$ & ,554 &,- 568 & 1 & & \\
\hline ATT &,$- 963^{* \prime \prime}$ &,- 811 &,$- 952^{\prime \prime \prime}$ & $\begin{array}{l}, 573 \\
\end{array}$ & ,951" &,- 570 & 697 & $\begin{array}{l}-, 304 \\
\end{array}$ &,$- 840^{\circ}$ & ,950" & $\begin{array}{l}-724 \\
\end{array}$ & 1 & \\
\hline IM & ,961" & $917^{\circ}$ & ,948" &,- 440 &,$- 990^{\prime \prime \prime}$ & ,310 &,- 681 & ,123 & ,931" &,$- 818^{\circ}$ & $\begin{array}{l}648 \\
, \quad 10\end{array}$ &,$- 941^{\prime \prime}$ & 1 \\
\hline
\end{tabular}




\section{Pérdida de peso}

Para efectos de la pérdida de peso del producto T1 y T2 (ver figura 2) presentaron una pérdida de peso día del 1,45 y 1,46 (\%) en contraste con 1,7 \% del TC. En términos de peso T1 y T2 no mostraron diferencias significativas, sin embargo, TC presentó una notoria perdida peso, coincidiendo con lo reportando en [5]. La pérdida del peso esta correlacionada de manera inversa con ATT hecho que concuerda con el coeficiente de correlación de Pearson para los tres tratamientos [6].
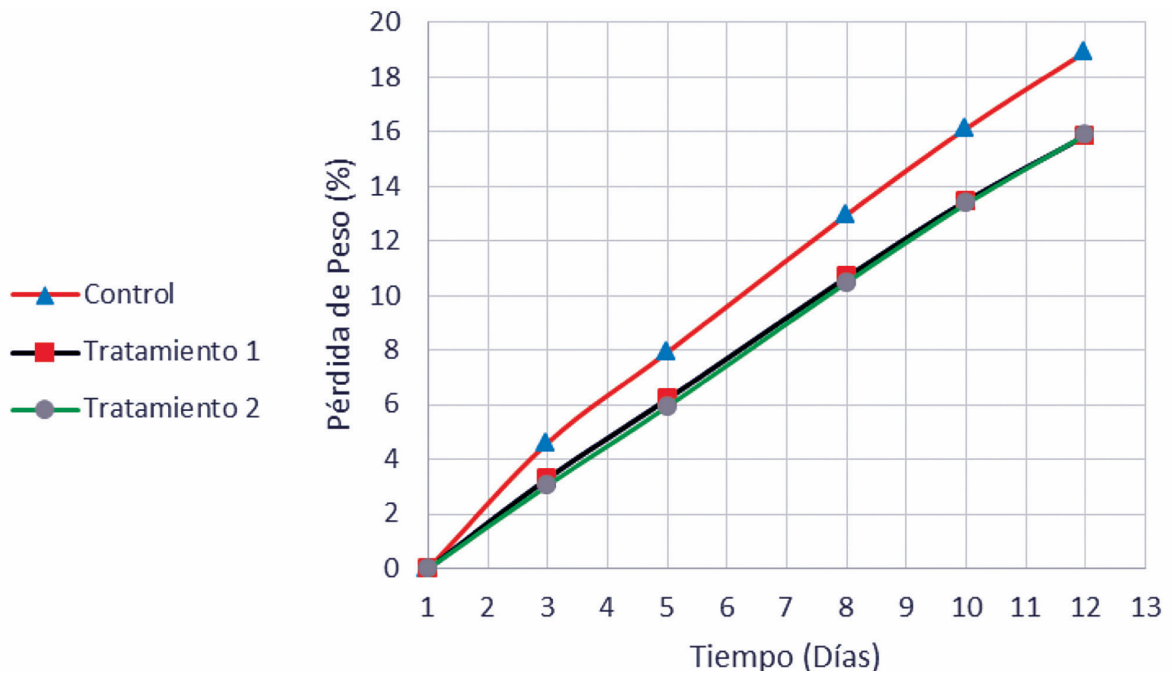

Figura 2. Porcentaje de pérdida de peso.

\section{IC externo}

En cuanto al color externo (ver figura 3) se encontró en la referencia [7], que los valores están entre -15,5 y -13,0; los cuales son próximos a los obtenidos en laboratorio y que oscilan entre -16,8 y -13,7. T1 tuvo diferencias en el índice de color que se pueden traducir en una degradación de la clorofila. Debido a las caracteristicas del producto el indice de color cambia dentro de los distintos tonos de verde [2].
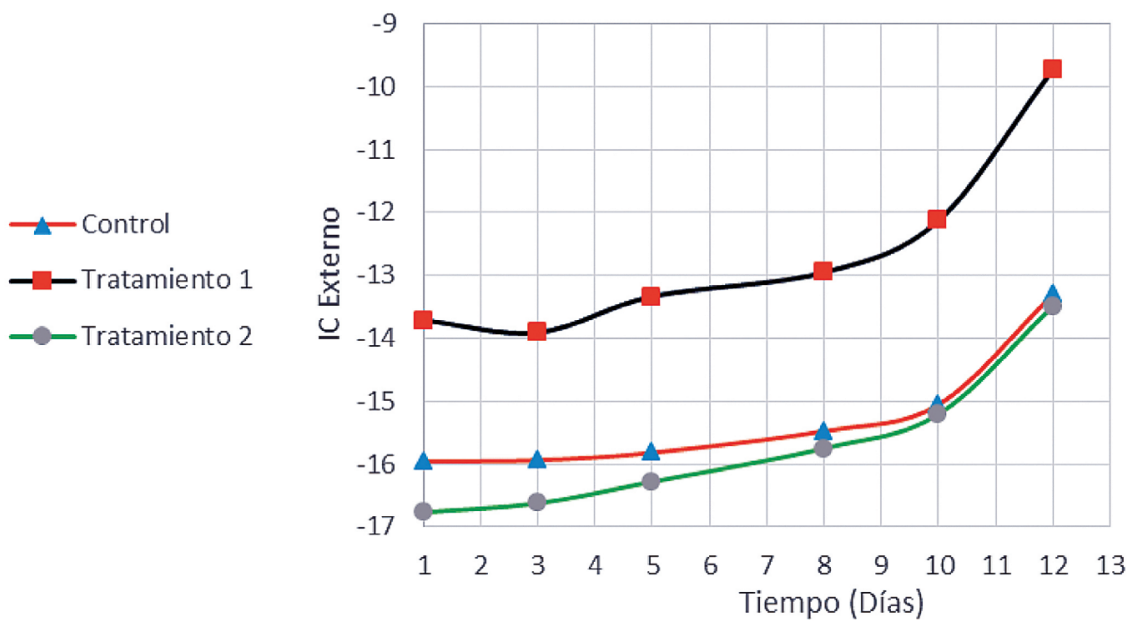

Figura 3. Índice de color externo. 


\section{Intensidad Respiratoria}

La guayaba es un producto que presenta producción de $\mathrm{CO}_{2}$ en concentraciones moderadas, con valores entre 70 y 150 (mg CO2/kg.h) [8]. En [2] se reporta una producción de $\mathrm{CO}_{2}$ entre 17,7 y 166,5 (mg CO2/kg.h). De igual manera, se encontró (figura 4) un rango no muy alejado que presenta una producción de CO2 entre 17,7 y 166,5 de mg CO2/kg.h; por lo cual, se puede inferir que este producto presenta altas concentraciones de $\mathrm{CO}_{2}$. En TC se presentó el pico climatérico más alto en comparación con T1 y T2.
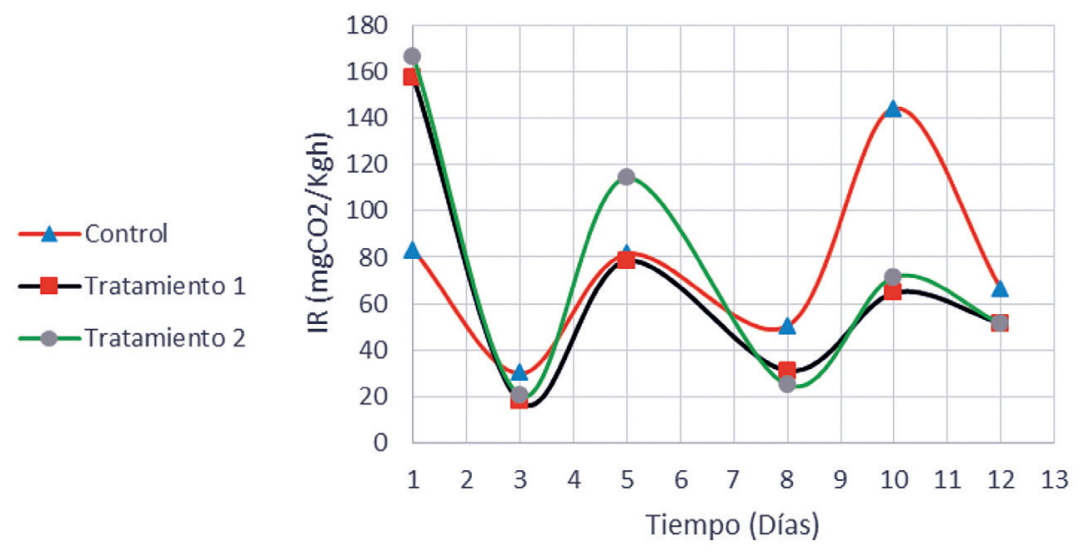

Figura 4. Intensidad respiratoria.

\section{Porcentaje de contracción del diámetro ecuatorial}

El diámetro ecuatorial se reduce en todos los tratamientos ya que tiene una relación directa con la pérdida de peso del fruto según la matriz de Pearson. El T2 mostró una tendencia lineal exceptuando el rango entre los días 8 y 12 que varió moderadamente, este tratamiento tuvo pérdidas diarias aproximadas del $0.5 \%$ (ver figura 5). El TC tuvo mayor porcentaje de contracción en comparación a los demás pero a partir del día 8 fue el único que presento una variación casi constante. En general T2 tuvo menor porcentaje de contracción que TC.

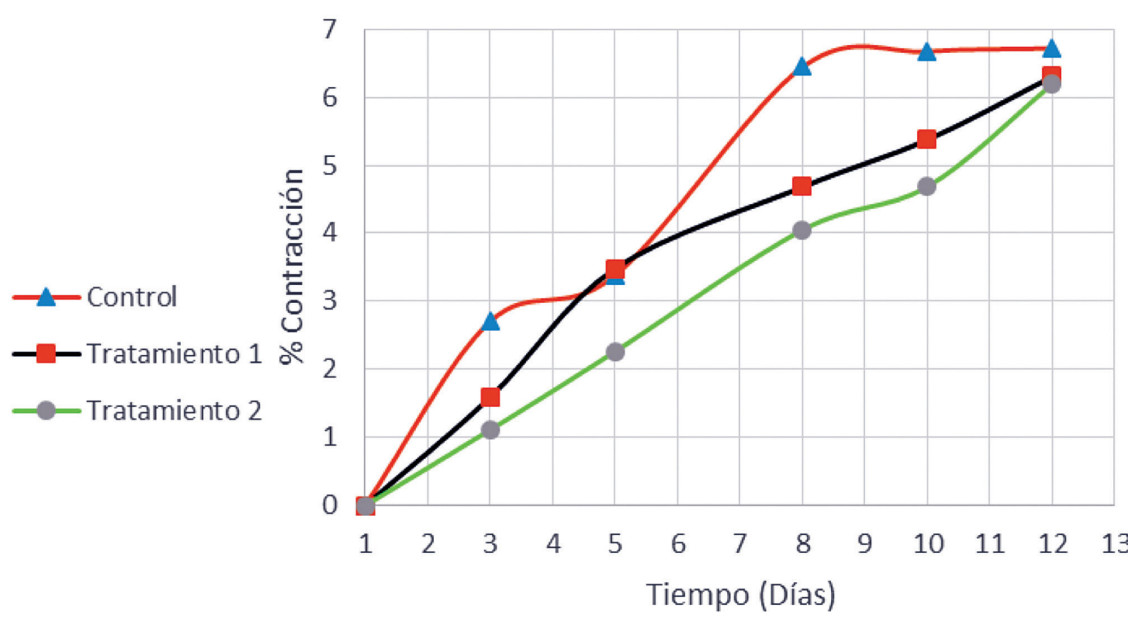

Figura 5. Porcentaje de contracción del diámetro ecuatorial. 


\section{Deformación}

Antes de la fractura viene la deformación y la referencia [9] indica que hay un tipo de fractura llamada frágil; la cual, empieza con una pequeña o nula deformación plástica que tiene una absorción baja de energía antes de que se dé la fractura.

En el texturómetro TC presento menores valores de deformación antes de que se fracturara el fruto por penetración, estos valores están entre 2,93 y 5,71 (mm), Entre 3,05 y 5,4 (mm) para T1; $3,97$ y $6,38(\mathrm{~mm})$ para T2 (ver figura 6$)$.
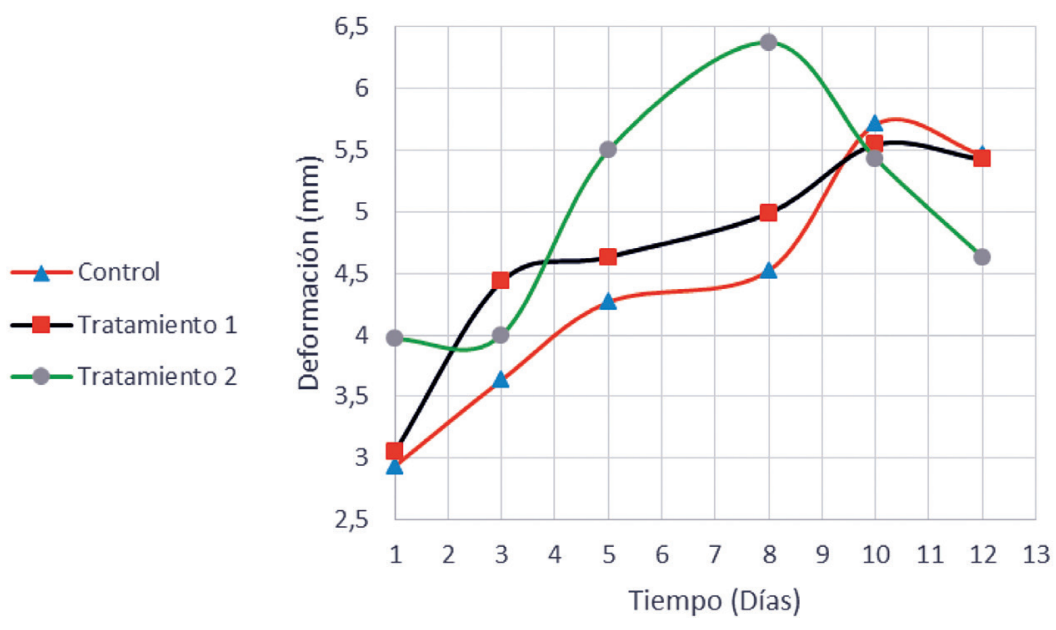

Figura 6. Deformación.

\section{Sólidos Solubles Totales}

Las variaciones en los grados 'Brix están asociadas a las tasas respiratorias y esto se puede enlazar con el tipo de comportamiento en TC y T1, sin embargo, la tendencia de T2 presentó una disminución constante de este parámetro [6] (ver figura 7). En [7] se reporta un rango de SST entre 10,84 y 11,45 ('Brix) el cual es cercano al rango 10,2 y 12,85 ('Brix) encontrado en esta investigación. Variedades colombianas de guayaba los SST aumentan con el avance del periodo poscosecha [10].
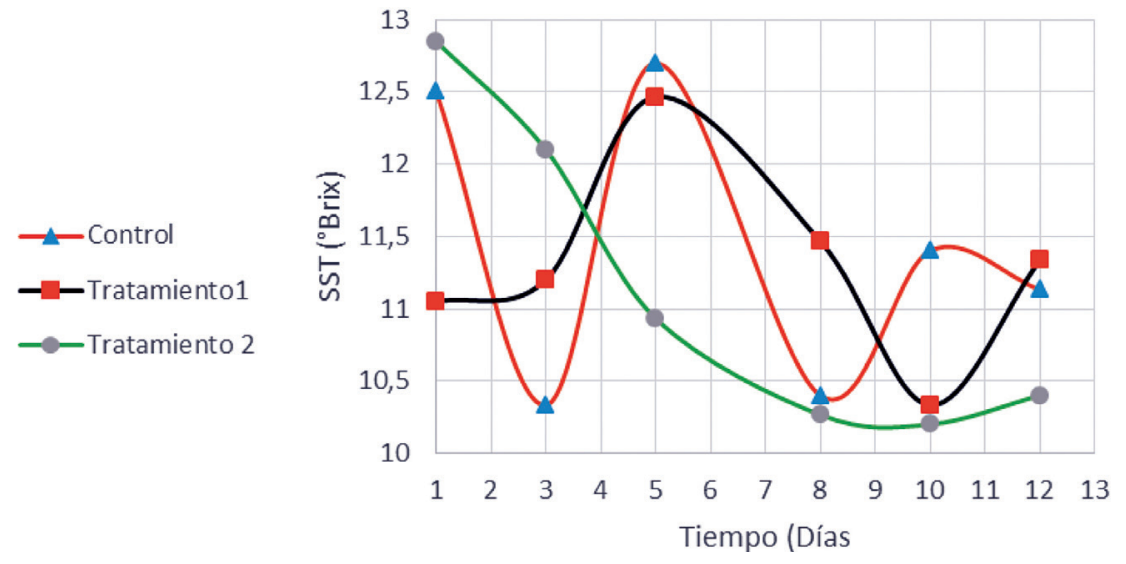

Figura 7. Solidos Solubles Totales. 
$\mathrm{pH}$

Para los tratamientos se encontró que el pH varia de 2,6 a 4,3 para TC, de 3,34 a 4,48 para T1 y de 3,2 a 4,1 para T2 (ver figura 8). A medida que se desarrolla el producto en poscosecha su $\mathrm{pH}$ va en aumento y autores reportan datos de $\mathrm{pH}$ entre 3,1 a 3,5 y 2,8 a 3,05 [2]. Aunque en [2] se afirma que la acidez total titulable no tiene una relación clara con el aumento de $\mathrm{pH}$; por el contrario, se encontró una relación inversamente proporcional entre estos dos parámetros según la matriz de Pearson ( $R^{2}=-88,6 \%$ para $T C, R^{2}=-87,9 \%$ para $T 1, R^{2}=-72,4 \%$ para $T 2$ ). El $\mathrm{pH}$ es un parámetro que ayuda a la estimación de los costos de transformación en procesos industriales [6].
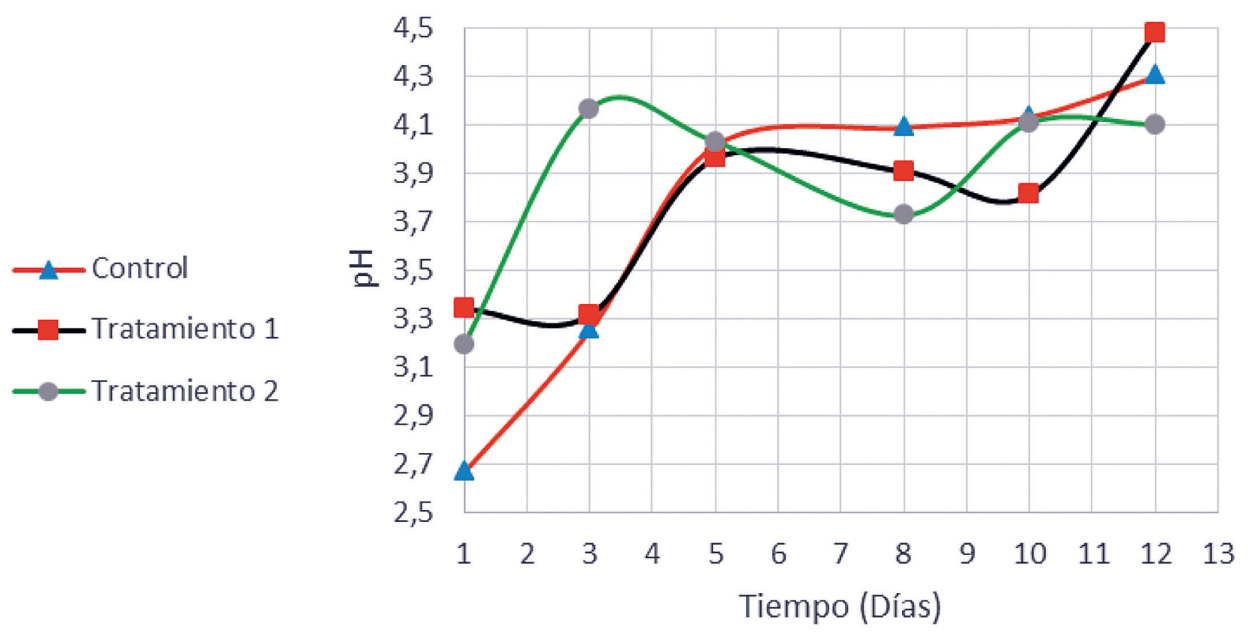

Figura 8. Potencial de Hidrógeno $(\mathrm{pH})$ en el transcurso de los días.

\section{Acidez Total Titulable}

Los parámetros ATT y pérdida de peso en la matriz de Pearson presentaron una relación inversamente proporcional muy evidente con valores de $-92,8 \%$ ** para TC, $-98,1 \%$ ** para T1 y $-96,3^{\star *}$ para T2, hecho que concuerda con lo reportado en [6], estudio que menciona que existe una correlación inversa entre ATT y la pérdida de peso, IR y los SST y además afirmando que la acidez se reduce en el proceso de maduración. Por el contrario, no se halla concordancia con los parámetros ATT contra la IR y SST, ya que la correlación entre ATT e IR es de: -9,8 \% para TC; 34,9 \% para T1 y 57,3 \% para T2; en el caso de la correlación ATT y SST es de 63,7 \% para TC, 20,9 \& para T1 y $95 \%$ ** para T2.

En la referencia [2][11], reportan que los frutos almacenados entre 4 a $23\left({ }^{\circ} \mathrm{C}\right)$ reducen la ATT entre 0,3 y 4,3 (\%). Hecho que se muestra la figura 9. Con tendencia a reducir los niveles de ATT en un rango entre 0,34 a 2,35 (\%).

\section{Índice de Madurez}

El grado de madurez de los frutos se infiere a través del IM. Los resultados de la figura 10, son evidencia de la relación SST/ATT. En T1 se mantiene un aumento progresivo hasta el día 10 y después presenta un proceso acelerado en el aumento del IM hasta el día 12. T2 muestra una tendencia de aumento moderado durante todo el periodo de tiempo. Se según la matriz de correlación de Pearson el IM es directamente proporcional al IC externo, esto quiere decir que 
a medida que el IM aumenta en todos los tratamientos se empieza a llevar a cabo un proceso de desverdizado, es decir, el cambio de color de la piel de verde oscuro a verde claro.

En la referencia [12] al aumentar la temperatura se promueve la maduración y por genética la fruta no cambia su color verde pero si cambia la tonalidad; de manera similar T1 y T2, almacenados a temperatura ambiente constante, el producto varia su tonalidad verde, este comportamiento pudo deberse al efecto de los tratamientos aplicados.
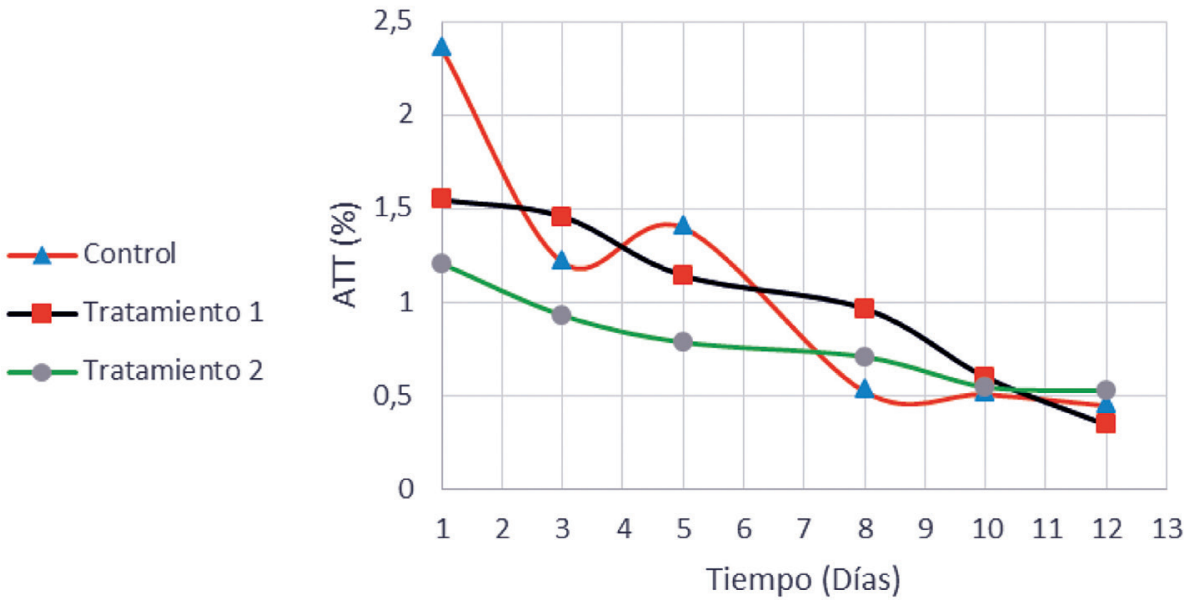

Figura 9. Acidez Total Titulable
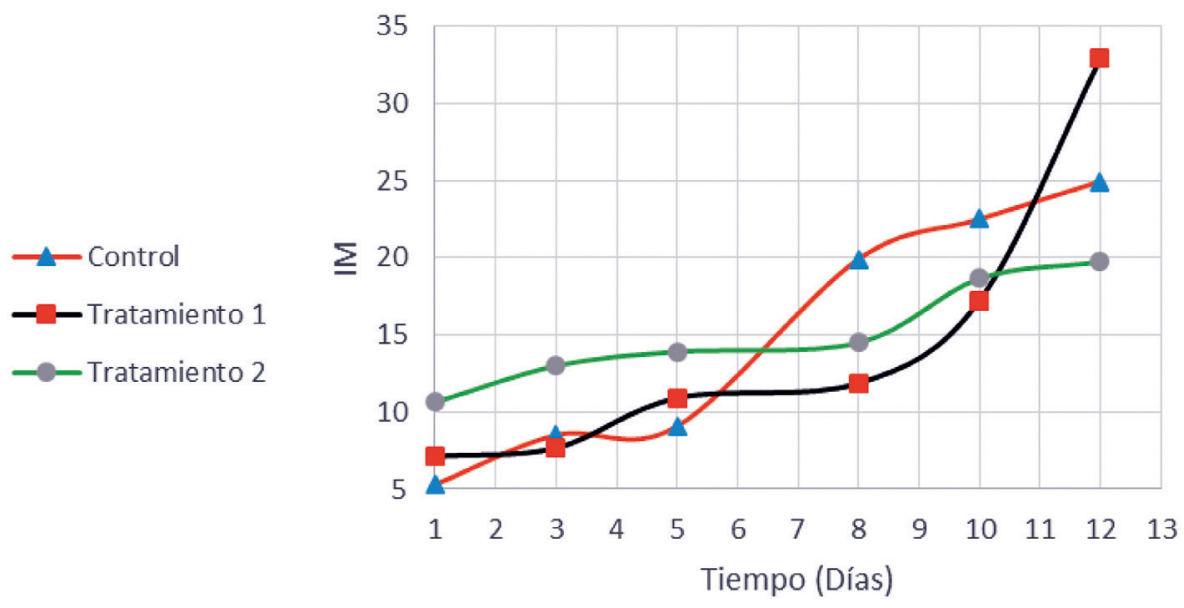

Figura 10. Índice de Madurez

\section{Conclusiones}

En general la muestra de los frutos de Feijoa del T2, que se sometieron a un proceso de humidificación con aire caliente a $41^{\circ} \mathrm{C}$ y $57 \%$ de humedad relativa durante 4 horas, mostraron resultados positivos en comparación con TC y T1. Revelando una menor reducción del porcentaje de pérdida de peso y consecuentemente en el porcentaje de contracción. Estos parámetros son importantes en la comercialización debido a que el peso del producto es un 
estimador de su precio. T2 también tuvo aumento moderado en el IM, los frutos soportaron mayores deformaciones antes de poder ser penetrados y presentó los menores valores de desverdización en el IC externo.

Se recomienda hacer un estudio de costos para implementar este proceso de humidificación en empresas que comercialicen Feijoa y también sé que se estudien estas tecnologías de poscosecha variando la temperatura de almacenamiento en refrigeración.

Se espera obtener mejores resultados al tener condiciones de aire caliente humidificado a mayor humedad relativa, lo que permitiría tener un mejor comportamiento de la pérdida de peso y las propiedades fisicoquímicas de los frutos de feijoa.

\section{Referencias}

[1] Frans Geilfus, El árbol al servicio del agricultor: Guía de especies - Frans Geilfus - Google Libros, vol. 2.1994.

[2] A. Parra and G. Fischer, "Maduración y comportamiento poscosecha de la feijoa (Acca sellowiana (O. Berg) Burret). Una revisión.," CIENCIAS HORTÍCOLAS, vol. 7, no. 1, pp. 98-110, 2013.

[3] Zhenzhong. L, Xu. L and Wang. Q, "Effect of hot air treatment on postharvest quality of zhonghuashou peach". Transactions of the Chinese Society of Agricultural Engineering, Volume 26, Number 1 pp. 375-379, 2010.

[4] ZHAO Yu-ying; LIU Le-cheng, "Effect of Hot Air Treatment on Storage Quality of Postharvest Cowpea Pod-Journal of Yangtze University (Natural Science Edition) 2015 15," J. Yangtze Univ. (Natural Sci. Ed., 2015).

[5] G. Soto-Zamora, E. M. Yahia, J. K. Brecht, and A. Gardea, "Effects of postharvest hot air treatments on the quality and antioxidant levels in tomato fruit," LWT - Food Sci. Technol., vol. 38, no. 6, pp. 657-663, Sep. 2005.

[6] M. Rodríguez, H. E. Arjona, and A. Galvis, "Maduración del fruto de feijoa (Acca sellowiana Berg) en los clones 41 (Quimba) y 8-4 a temperatura ambiente en condiciones de la Sabana de Bogotá," Agron. Colomb., vol. 24, no. 1, pp. 68-76, 2006.

[7] R. R. Martínez-Vega, G. Fischer, A. Herrera, B. Chaves, and O. C. Quintero, "Características físico-químicas de frutos de feijoa influenciadas por la posición en el canopi," Rev. Colomb. Ciencias Hortícolas, vol. 2, no. 1, pp. 21-32, Feb. 2011.

[8] A. A. Kader and E. M. Yahia, "Postharvest biology of tropical and subtropical fruits," in Postharvest Biology and Technology of Tropical and Subtropical Fruits, vol. 1, Elsevier Ltd., pp. 79-111, 2011

[9] M. Bourne, Food Texture \& viscosity. Concept and Measuremente. Academic Press, pp. 427, 2002.

[10] M. E. Solarte, M. Soledad Hernández, A. L. Morales, J. P. Fernández-Trujillo, and L. M. Melgarejo, “Caracterización Fisiológica Y Bioquímica Del Fruto De Guayaba Durante La Maduración," pp. 85-119, 2010.

[11] A. C. Velho, C. V. T. do Amarante, L. C. Argenta, and A. C. Steffens, "Influência da temperatura de armazenamento na qualidade pós-colheita de goiabas serranas," Rev. Bras. Frutic., vol. 33, no. 1, pp. 14-20, Mar. 2011.

[12] C. V. T. do Amarante, C. A. Steffens, J. P. H. J. Ducroquet, and A. Sasso, "Qualidade de goiaba-serrana em resposta à temperatura de armazenamento e ao tratamento com 1-metilciclopropeno," Pesqui. Agropecu. Bras., vol. 43, no. 12, pp. 1683-1689, Dec. 2008. 